
(2015)

\title{
INFLUÊNCIA DA TOPOGRAFIA NA MOLHABILIDADE EM SUPERFÍCIES DE TITÂNIO TRATADAS POR PLASMA
}

\author{
Marco Aurélio Medeiros da Silva \\ Universidade Federal do Rio Grande do Norte, Programa de Pós-Graduação do CCSA, \\ Campus Universitário, Lagoa Nova, Natal, RN, Brasil. CEP: 59072-970. \\ marcoaureliobucofacial@yahoo.com.br \\ Paulo Victor de Azevedo Guerra
}

Universidade Federal do Rio Grande do Norte, Departamento de Engenharia Biomédica, Lagoa Nova, Natal, RN, Brasil. CEP: 59072-970.

paulovictorguerra@gmail.com

Cleophatra Aquino Fernandes

Universidade Federal do Rio Grande do Norte, Departamento de Odontologia, Campus

Universitário, Lagoa Nova, Natal, RN, Brasil. CEP: 59072-970.

Cleophatrafernandes@yahoo.com.br

Ricardo Alexsandro de Medeiros Valentim

Universidade Federal do Rio Grande do Norte, Departamento de Engenharia Biomédica,

Campus Universitário, Lagoa Nova, Natal, RN, Brasil. CEP: 59072-970.

ricardo.valentim@ufrnet.br

\section{Karilany Dantas Coutinho}

Universidade Federal do Rio Grande do Norte, Departamento de Engenharia Biomédica, Campus Universitário, Lagoa Nova, Natal, RN, Brasil. CEP: 59072-970.

karilany@ufrnet.br

\section{Clodomiro Alves Junior}

Universidade Federal Rural do Semi-Árido, Departamento de Física,

Costa e Silva, Mossoró - RN, 59625-900.

clodomiro.jr@gmail.com

Custódio Leopoldino de Brito Guerra Neto

Universidade Federal do Rio Grande do Norte, Departamento de Engenharia Biomédica,

Campus Universitário, Lagoa Nova, Natal, RN, Brasil. CEP: 59072-970.

custodioguerra@yahoo.com.br

\section{RESUMO}

O titânio, devido sua afinidade com o oxigênio, possui uma camada óxida crescida espontaneamente durante as diferentes etapas de seu processamento. Para eliminar essa camada durante o pré-tratamento a plasma, geralmente utiliza-se um pré-tratamento para limpeza. Com o objetivo de estudar a influência topográfica na molhabilidade de superfícies tratadas por plasma, utilizou-se atmosferas de $\mathrm{Ar}, \mathrm{H}_{2}$ e mistura $\mathrm{Ar}-\mathrm{H}_{2}$. Foram utilizados 18 discos de Ti cp (grau II), com $10 \mathrm{~mm}$ de diâmetro e $1 \mathrm{~mm}$ de espessura. Estas amostras foram submetidas durante 20 e 60 minutos num plasma produzido por uma descarga de cátodo oco (HCD), por uma voltagem de $500 \mathrm{~V}$, atmosferas de $\mathrm{Ar}, \mathrm{H}_{2}$ e $\mathrm{Ar} / \mathrm{H}_{2}$ e pressão de $220 \mathrm{~Pa}$. Nessas condições a temperatura das amostras elevou-se a 473K. Após o pré-tratamento, o estado das superfícies das amostras foi avaliado quanto às fases superficiais (GIDRX), nanotopografia (AFM) e molhabilidade (ensaio da gota séssil). Verificou-se que todas as condições foram efetivas na redução do óxido e que resultaram em diferentes valores de molhabilidade. Avaliou-se a correlação entre os parâmetros 

(2015)

topográficos $\mathrm{Ra}$ e $\mathrm{Rp} / \mathrm{Rz}$ com a molhabilidade para as diferentes condições de tratamento. Diante dos resultados obtidos conclui-se que os parâmetros $\mathrm{Ra} e \mathrm{Rp} / \mathrm{Rz}$ não são apropriados para correlacionar com a molhabilidade e sugere-se um novo parâmetro topográfico que contemple a equação de Cassie - Baxter.

PALAVRAS-CHAVE: Titânio; Tratamento a plasma; Biomateriais; Redução de óxido de titânio; Molhabilidade; Rugosidade.

\title{
INFLUENCE OF TOPOGRAPHY ON PLASMA TREATED TITANIUM SURFACE WETTABILITY
}

\begin{abstract}
With the aim of studying the topographic influence on wettability of plasma-treated surfaces, we used eighteen commercially pure titanium (CP Titanium) disks (grade II), 10 $\mathrm{mm}$ in diameter and $1 \mathrm{~mm}$ thick, and in atmospheres of Argon (Ar), Hydrogen H2and Ar$\mathrm{H}$. These samples were submitted to plasma produced by hollow cathode discharge (HCD) for 20 and $60 \mathrm{~min}$ at a voltage of $500 \mathrm{~V}$ and pressure of $220 \mathrm{~Pa}$. After pre-treatment, the state of the sample surfaces was assessed for surface phases with grazing incidence X-ray diffraction (GIXRD), and XPS (X-ray photoelectron spectroscopy), evaluated by atomic force microscopy (AFM) and wettability, using the sessile drop test. It was found that all the conditions were effective in reducing oxide, resulting in different wettability values. Correlation between average roughness ( $\mathrm{Ra}$ ) parameters and the ratio between maximum peak height and average distance between the highest peak and lowest valley $(\mathrm{Rp} / \mathrm{Rz})$ and wettability for the different treatment conditions were analyzed. Given the results obtained, it was concluded that $\mathrm{Ra}$ and $\mathrm{Rp} / \mathrm{Rz}$ are not appropriate for correlating with wettability and it is suggested that a new topographic parameter be adopted for the Cassie-Baxter equation. XPS analysis showed that reduction efficacy was greater for the $\mathrm{Ar}-\mathrm{H}$ mixture, followed by Ar and $\mathrm{H} 2$.
\end{abstract}

\section{KEYWORDS:}

Titanium; Plasma treatment; Biomaterials, Titanium oxide reduction; Wettability; Roughness parameter. 


\section{INFLUÊNCIA DA TOPOGRAFIA NA MOLHABILIDADE EM SUPERFÍCIES DE TITÂNIO TRATADAS POR PLASMA}

\section{INTRODUÇÃO}

Hoje o material mais utilizado em aplicações biomédicas é o titânio e suas ligas, devido sua alta biocompatibilidade e resistência à corrosão ${ }^{1}$. Entretanto técnicas de tratamento e revestimento de superfícies são utilizadas para adaptá-los às várias aplicações. $\mathrm{Na}$ engenharia tecidual, por exemplo, há um crescente interesse em se desenvolver uma nova geração de materiais que, além de biocompatíveis e resistentes à corrosão, tenham boa molhabilidade para garantir uma maior integridade da interface osso/biomaterial ${ }^{2}$. Ao contrário disso, em stents e válvulas cardíacas há necessidade que o sangue tenha baixa molhabilidade para evitar adesão plaquetária ${ }^{2}$. Esses dois exemplos extremos ilustram bem a necessidade da modificação de superfície após a fabricação do dispositivo biomédico. De uma maneira geral, a fabricação desses dispositivos compreende uma série de etapas como usinagem, estampagem, dobramento e/ou tratamentos térmicos que expõem a superfície do titânio aos mais variados contaminantes orgânicos e inorgânicos. Nesse sentido, após a fabricação há necessidade de procedimentos que incluem protocolos de limpeza e passivação ${ }^{3}$. Esses procedimentos são recomendados por órgãos que estabelecem normas e condutas técnicas para fabricação de materiais para uso biológico, com a finalidade de obter superfícies que possam ter melhor interação com os tecidos onde são implantados ${ }^{4}$. Dentre os métodos utilizados podem-se citar os mecânicos que são realizados à custa de jateamento no substrato com partículas de diversas granulações; método químico que utiliza banhos sucessivos de ácidos, tratamento alcalino, peróxido de hidrogênio, sol-gel e oxidação anódica (decapagem) e os métodos físicos que utilizam o laser, spray térmico, plasma spray, PVD, implantação iônica, implantação de íons e plasma ${ }^{5}$. O método químico é o mais utilizado, limpa e trata a superfície, porém poderá levar à incorporação de elementos nocivos ao material e tornar necessárias etapas adicionais para descontaminação, aumentando tempo e custo a esse processo, além de causar problemas no descarte de resíduos ao meio ambiente, após o seu uso, ocasionando danos de difícil reparação para o mesmo ${ }^{6}$.

O plasma tem sido bem requisitado no processamento de superfície na indústria de microeletrônicos e tem atraído à atenção na área de biomateriais ${ }^{7}$. Embora esse método tenha sido usado vastamente em pesquisa de biomateriais, as informações são limitadas sobre os parâmetros do processo.

Uma das vantagens desse método está na versatilidade da energia do plasma. Num mesmo equipamento é possível limpar, modificar uma superfície e esterilizá-la. Na indústria biomédica esse processo consiste em modificar a superfície em uma atmosfera altamente ionizada. Quando se deseja uma atmosfera mais hostil utiliza-se a configuração cátodo oco. A alta ionização é conseguida porque os elétrons do plasma são obrigados a refletirem sucessivamente entre duas superfícies catódicas, onde os elétrons são repelidos pelo cátodo central que pode ser a peça que se deseja oxidar em direção aos cátodos externos. Ao se aproximarem destes, são também repelidos, realizando assim, um movimento de zig-zag que aumentará a taxa de ionização da região em questão. Uma alta densidade de íons significará maior bombardeamento na superfície, ocasionando maior incidência energética ${ }^{7,8,9}$. Esta configuração de eletrodos é utilizada quando se deseja aumentar o dano superficial, ou seja, para uma mesma 
temperatura de processo, produzem-se mais defeitos na superfície que na oxidação com a descarga planar ${ }^{10}$.

Alguns autores têm investigado o tratamento em descarga planar para prétratamento de superfícies e/ou modificações de biomateriais metálicos de titânio ${ }^{5}$. Foi reportado também que uma mistura gasosa de plasma $\mathrm{H}_{2} / \mathrm{Ar}$, pode ser eficiente na remoção de óxidos metálicos em um pré-tratamento, onde radicais de hidrogênio pode ajudar a reduzir óxidos, enquanto que o argônio atua aumentando a eficiência da taxa de ionização ${ }^{11}$.

Segundo Zhu et al, é importante melhorar aspecto nanotopográficos, químicos e físicos visando o aumento da molhabilidade e sítios de adesão celular ${ }^{8,12,13}$. Baseado nesta afirmação e em resultados conflitantes da literatura acerca da correlação entre os aspectos físico-químicos da superfície com a molhabilidade, adotamos uma sistemática de limpeza de superfícies de titânio para analisar sua influência sobre a molhabilidade ${ }^{7}$, 10,14, O presente trabalho tem por objetivo estudar a influência da topografia na molhabilidade de superfícies tratadas por plasma em atmosferas de $\mathrm{Ar}$ e $\mathrm{H}_{2}$ e diferentes tempos de tratamento.

\section{MATERIAIS E MÉTODOS}

O processo de limpeza por plasma foi realizado numa câmara cilíndrica de aço inoxidável, com $400 \mathrm{~mm}$ de diâmetro e $400 \mathrm{~mm}$ de comprimento, hermeticamente fechada por dois flanges (Figura 1). No flange inferior se encontram as entradas para gás, bomba de vácuo, sensor de pressão e eletrodo catodicamente polarizado. No centro do eletrodo está inserido axialmente um termopar, com o qual se mede a temperatura da amostra durante o experimento. Foi utilizado termopar do tipo K (cromel-alumel). A pressão interna da câmara foi medida através de sensor de membrana capacitiva, tipo baratron, Edwards.

Internamente a câmara possui um porta amostra em forma de disco, sobre o qual é colocada a amostra. Na configuração de cátodo oco a amostra está contida no interior de um recipiente conforme ilustrado na figura $1 \mathrm{~b}$. Fixou-se a distância entre cátodos (distância entre a superfície da amostra e a tampa superior - $\mathrm{d}_{\mathrm{c}-\mathrm{c}}$ ), em $9 \mathrm{~mm}$.

Uma fonte de corrente continua, com tensão de saída continuamente ajustável até $1200 \mathrm{~V}$ e corrente máxima de 1,5 A, foi utilizada para alimentar a descarga. Além da câmara e da fonte de tensão, há também o sistema de vácuo e distribuição de gases que completam assim o equipamento.

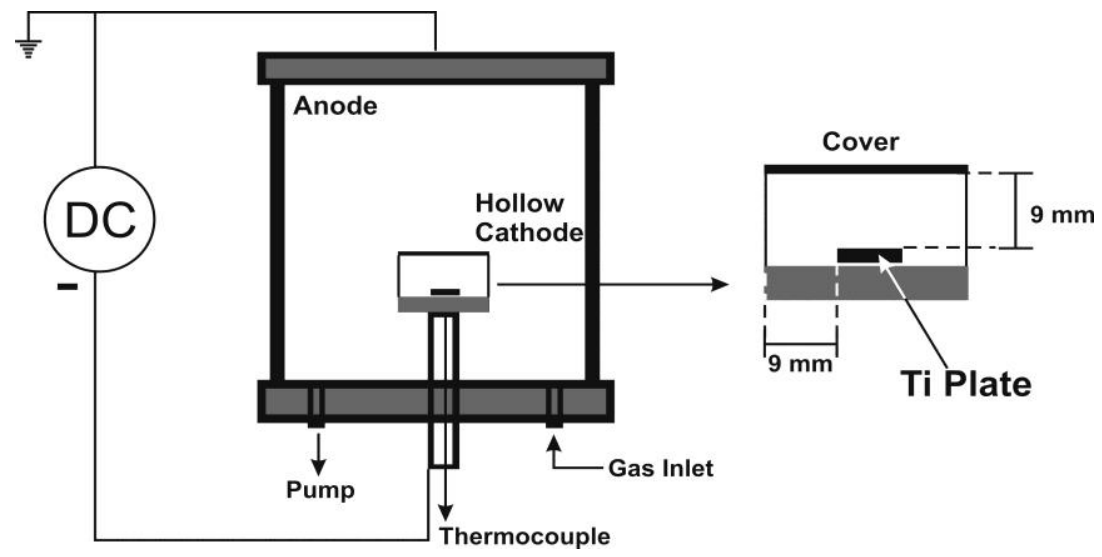

Figura 1 - Diagrama esquemático do reator a plasma e periféricos. 
As condições de pré-tratamentos estão ilustradas Tab. 1. Para a limpeza por plasma, inicialmente a câmara foi evacuada até uma pressão na faixa de $10 \mathrm{~Pa}$ e então introduzido o gás de trabalho até a pressão de $220 \mathrm{~Pa}$. Neste instante a fonte era ligada e ajustada até uma tensão de $500 \mathrm{~V}$, quando então o gás começava a brilhar. Ajustada a pressão, esperava-se que a amostra atingisse a temperatura desejada (473K) para a limpeza. Após os tratamentos, os 18 discos (3 discos para cada condição) foram submetidos às análises por difração de raios-X com ângulo rasante (GIDRX), microscopia de força atômica (AFM) e ensaio de molhabilidade (gota séssil).

Tab.1 Condição de pré-tratamento, gás utilizado, tempo.

\begin{tabular}{l|l|c} 
Amostra & Atmosfera & $\begin{array}{c}\text { Tempo } \\
\text { (Min) }\end{array}$ \\
\hline Ar 20 & Pure Ar & 20 \\
Ar 60 & Pure Ar & 60 \\
$\mathrm{H}_{2} 20$ & Pure hidrogen & 20 \\
$\mathrm{H}_{2} 60$ & Pure hidrogen & 60 \\
$\mathrm{Ar}+\mathrm{H}_{2} 20$ & $50 \% \mathrm{Ar}+50 \% \mathrm{H}_{2}$ & 20 \\
$\mathrm{Ar}+\mathrm{H}_{2} 60$ & $50 \% \mathrm{Ar}+50 \% \mathrm{H}_{2}$ & 60
\end{tabular}

A fase e cristalinidade da superfície foi analisada usando difração de raios- $X$ em ângulo rasante (GIDRX), com incidência igual a $0,5^{\circ}$. O equipamento utilizado foi um difratômetro modelo Shimadzu XRD-6000. Os espectros foram obtidos usando radiação $\mathrm{Cu} K \alpha$ (comprimento de onda: 0,154 nm), operado em $40 \mathrm{KV}$, no intervalo entre $20^{\circ}$ e $90^{\circ}$. As identificações dos picos dos difratogramas foram realizadas com auxílio das tabelas de cristalografia padrão JCPDS.

Um dos estudos que compõe a análise de molhabilidade é a determinação do ângulo de contato através da técnica da gota séssil. As amostras, antes e após o tratamento, são colocadas num goniômetro construído no Laboratório de Processamento de Materiais por Plasma (LABPLASMA) (Figura 2) e em seguida, utilizando uma micropipeta regulada para $20 \mu 1$, uma gota de água destilada é liberada sobre a amostra e sua imagem captada pela câmera de vídeo do goniômetro. Com o uso do programa suftens (programa também desenvolvido no LABPLASMA) são determinados os valores dos ângulos de contato. $\mathrm{O}$ processo é repetido com o glicerol, que possui componente polar e apolar contrária à água.

Foram realizadas três medidas em cada amostra, significando que para cada condição estudada eram utilizados 09 valores para cada líquido. O objetivo é observar o comportamento da gota sobre a superfície da amostra durante 60s. 


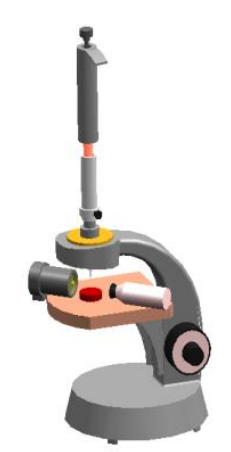

Figura 2 - Aparelho desenvolvido no laboratório para medir o espalhamento da gota sobre as amostras.

Para análise da nanotopografia da superfície foi utilizado um microscópio de força atômica da SHIMADZU modelo SPM 9600. Através da imagem obtida foi possível determinar os diferentes parâmetros de rugosidade. Obteve-se imagens num quadrado com área de 5 x 5 micron $^{2}$.

\section{RESULTADOS E DISCUSSÕES}

Na figura 3 são apresentados os espectros de difração de raios-X em ângulo rasante das amostras tratadas e também da amostra sem tratamento. Todas as condições de tratamento resultaram numa redução do $\mathrm{TiO}_{2}$ para o $\mathrm{Ti}_{2} \mathrm{O}$, indicando que as seis condições utilizadas foram eficazes na redução dos teores de oxigênio. No presente trabalho observase que não apenas o $\mathrm{H}_{2}$ é efetivo na remoção dos óxidos, mas também o Ar ou misturas desses são eficazes. $\mathrm{Na}$ verdade, a atmosfera com $\mathrm{H}_{2}$ puro mostrou ser o mais eficaz para tempo de tratamento de 60 minutos, conforme pode ser visto na figura $3 \mathrm{~b}$. Nesta condição verifica-se que a razão de intensidade do pico referente à fase $\mathrm{Ti}_{2} \mathrm{O}$ é muito pequena comparativamente à intensidade do pico Tí (101). Para as demais condições pode-se afirmar que não houve diferenças significativas nas razões desses picos.

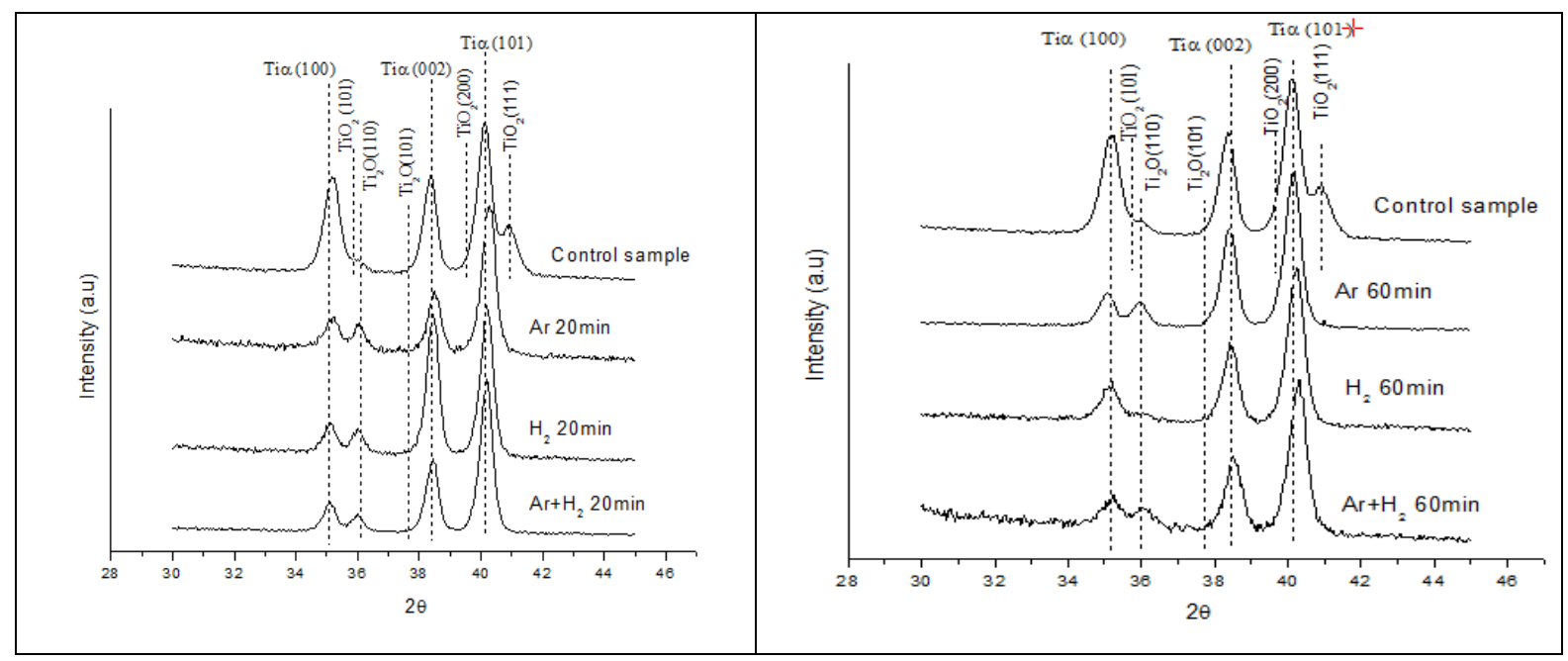

Figura 3 - Difração de Raio-X de amostras tratadas em plasma comparativamente com amostra não tratada com incidência rasante de 0,5 grau. 

(2015)

Pode-se então afirmar que as diferenças entre as superfícies, se houver, devem ser de origem topográfica. Sabendo que a molhabilidade da superfície é resultante de fatores físicos e químicos ${ }^{2}$, espera-se que os resultados da topografia da superfície possam responder pelos diferentes valores de molhabilidade que serão verificados nesse trabalho, uma vez que quimicamente foi verificado não haver diferenças entre as superfícies, excetuando apenas a amostra $\mathrm{H}_{2} 60$ que apresentou a menor intensidade do pico de $\mathrm{Ti}_{2} \mathrm{O}$.

A nanotopografia tem sido definida como uma superfície que apresenta topografia característica, com uma magnitude de $100 \mathrm{~nm}$ ou menos. Essas modificações alteram as interações da superfície com íons, biomoléculas e células que influenciam na adesão, proliferação e diferenciação celular, que vão interferir no processo de osseointegração, potencializando seus efeitos ${ }^{15}$.

Em todas as amostras tratadas foi verificada modificação topográfica. Na figura 4 são apresentadas ilustrativamente uma superfície do titânio polido e outra após tratamento por plasma (tratada). Verifica-se que a amostra tratada com $\mathrm{Ar} / \mathrm{H}_{2} 20$ minutos apresenta uma superfície modificada, comparativamente à amostra controle, devido ao bombardeamento iônico causado pelo argônio e/ou hidrogênio. Ainda na mesma amostra, pode-se explorar alguns parâmetros de rugosidade que serão importantes na avaliação da molhabilidade das amostras. Na figura 4 são apresentados os valores dos picos de maior altura para as duas superfícies. Além disso, são fornecidos pelo programa computacional outros parâmetros como $\mathrm{Ra}, \mathrm{Rp}, \mathrm{Rv}$ e $\mathrm{Rz}$, que são extraídos das curvas de perfis apresentados ao lado de cada figura.

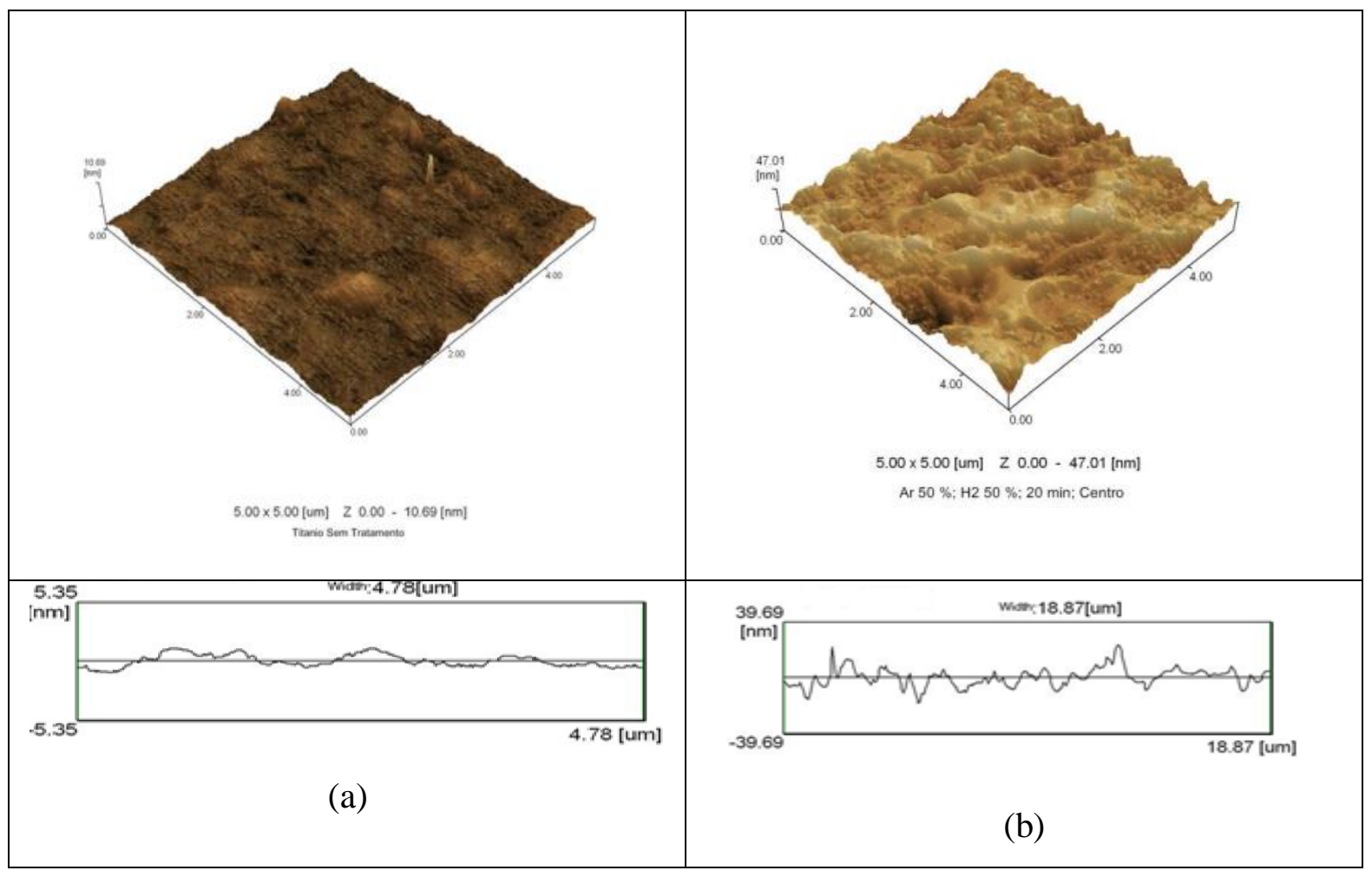

Figura 4 - Nanotopografias das superfícies (a) amostra polida, (b) amostra tratada em mistura de Ar e $\mathrm{H}_{2}$ durante 20 minutos $\left(\mathrm{Ar}+\mathrm{H}_{2} 20\right)$

O parâmetro Ra é definido como a altura média de picos e vales existente numa superfície ${ }^{16}$. Uma alteração nesse parâmetro não expressa todas as variações de 
rugosidade, ou seja, alterações na distribuição e alturas dos picos podem compartilhar valores de (Ra) em comum, não definindo com clareza o comportamento do relevo superficial $^{15}$. Vários autores correlacionam os valores de Ra com a molhabilidade da superfície ${ }^{15,16,17}$. Todos afirmam que existe uma relação direta entre a rugosidade ( $\left.\mathrm{Ra}\right)$ e a molhabilidade.

Outra opção de expressar o perfil topográfico seria com a utilização dos parâmetros Rp e Rz. O parâmetro Rp é obtido pela média de altura do pico em relação à linha central em 5 leituras consecutivas e o Rz é calculado pela soma da altura máxima dos picos (Rp) com a profundidade máxima dos vales $(\mathrm{Rv})$, no comprimento da amostragem ${ }^{12}$. A razão $\mathrm{Rp} / \mathrm{Rz}$ é de especial importância na avaliação do formato da superfície, pois uma razão maior que 0,5 indica picos pontiagudos enquanto valores menores que 0,5 indica uma superfície com picos arredondados ${ }^{12}$. Segundo esses autores, picos arredondados favorecem o espalhamento de líquidos na superfície. Usando essa hipótese, através da avaliação da razão $\mathrm{Rp} / \mathrm{Rz}$ pode-se estimar sua influência sobre a molhabilidade ${ }^{12}$. A análise da molhabilidade é um fator importante para definir a biocompatibilidade e essa por sua vez tem importância primordial na osseointegração dos biomateriais. ${ }^{18,13}$ Ângulos de contatos baixos significam maior molhabilidade, isto é, superfície mais hidrofílica ${ }^{2}$.

No presente trabalho foram obtidos os valores de Ra, Rp e Rz a partir da média obtida em 2 perfis de níveis traçados para cada superfície observada. Também foram medidos os ângulos de contato de gotas séssil de água destilada e glicerol, quando depositadas sobre essas superfícies. Os resultados estão apresentados na tabela 2.

Tabela 2 - Valores de rugosidade e ângulo de contato

\begin{tabular}{|c|c|c|c|c|c|c|}
\hline Amostras & $\mathbf{R a}(\mathbf{n m})$ & $\underset{(\mathbf{n m})}{\mathbf{R z}}$ & $\underset{(\mathbf{n m})}{\mathbf{R p}}$ & $\mathbf{R p} / \mathbf{R z}$ & $\begin{array}{c}\begin{array}{c}\text { Ângulo de } \\
\text { contato }\left(\mathrm{H}_{2} \mathrm{O}\right)\end{array} \\
(\theta) \\
\end{array}$ & $\begin{array}{c}\text { Ângulo de } \\
\text { contato } \\
\text { (Glicerol) } \\
(\theta) \\
\end{array}$ \\
\hline Controle & 0,51 & 10,67 & 0,68 & 0,063730084 & 52,67 & 52,90 \\
\hline $\operatorname{Ar}(20)$ & 6,57 & 59,6 & 37,4 & 0,627516779 & 48,71 & 53,00 \\
\hline $\operatorname{Ar}(60)$ & 8,53 & 93,08 & 60,23 & 0,647077783 & 56,8 & 64,00 \\
\hline $\mathrm{H}_{2}(20)$ & 4,9 & 85,34 & 62,35 & 0,730606984 & 89,87 & 86,30 \\
\hline $\mathrm{H}_{2}(60)$ & 6,3 & 53,89 & 24,7 & 0,458341065 & 44,69 & 80,50 \\
\hline $\mathrm{Ar} / \mathrm{H}_{2}(20)$ & 4,5 & 46,94 & 21,3 & 0,453770771 & 38,64 & 59,60 \\
\hline $\mathrm{Ar} / \mathrm{H}_{2}(60)$ & 5,64 & 41,43 & 19,99 & 0,482500603 & 63,16 & 72,40 \\
\hline
\end{tabular}

No presente trabalho, a correlação direta de Ra com a molhabilidade não foi observada nem mesmo entre pares de amostras tratadas em tempos diferentes. Como pode ser observado na tabela 2, houve aumento da rugosidade Ra para todas as condições quando foi aumentado o tempo de bombardeamento iônico. Por exemplo, a amostra bombardeada com argônio durante 20 min. (Ar 20) aumentou o Ra de 59,6 nm para 93,1 $\mathrm{nm}$, quando bombardeada durante 60 minutos (Ar 60). Entretanto, quando se observa os ângulos de contato não houve aumento da molhabilidade, como previsto na literatura ${ }^{19}$. Como pode ser observado o ângulo de contato aumentou o que significa decréscimo de molhabilidade. O mesmo acontece com o $\mathrm{Ar} / \mathrm{H}_{2} 20 \mathrm{~min}$ e $60 \mathrm{~min}$, a rugosidade aumenta enquanto a molhabilidade diminui. Apenas para as amostras tratadas com $\mathrm{H}_{2}$ é que foi observada uma correlação direta entre o parâmetro Ra e a molhabilidade. 
Observando agora na tabela 2 a correlação entre a razão $\mathrm{Rp} / \mathrm{Rz}$ para pares de amostras tratadas na mesma atmosfera, mas em tempos diferentes, verifica-se que ela existe apesar de não ter uma proporcionalidade direta. Para amostras tratadas na atmosfera de $\mathrm{H}_{2}$ puro, pode ser observado que os picos passaram de pontiagudos (razão $\mathrm{Rp} / \mathrm{Rz}$ maior que 0,5 ) quando tratadas durante 20 minutos, para picos arredondados (razão $\mathrm{Rp} / \mathrm{Rz}$ menor que 0,5$)$ quando tratadas durante 60 minutos. De fato, a molhabilidade é maior nos picos arredondados, conforme previsto na literatura. Para os demais pares, as diferenças na razão $\mathrm{Rp} / \mathrm{Rz}$ não são tão grandes como nas amostras tratadas com $\mathrm{H}_{2}$. Observa-se que as amostras tratadas durante 60 minutos em argônio possuem os picos mais pontiagudos, enquanto nas amostras tratadas com a mistura $\mathrm{Ar}+\mathrm{H}_{2}$ possuem picos mais arredondados.

Para as amostras tratadas com a mistura $\mathrm{Ar}+\mathrm{H}_{2}$, embora os resultados da razão $\mathrm{Rp} / \mathrm{Rz}$ sejam muito próximos, observa-se que os valores das molhabilidades são muito diferentes, indicando pouca ou nenhuma correlação.

Na figura 5 são apresentados os valores dos ângulos de contato de uma gota séssil de água destilada (5a) e glicerol (5b) para todas as amostras estudadas. De uma maneira geral, houve pouca variação entre os valores dos ângulos de contatos quando se mudou da água para o glicerol.

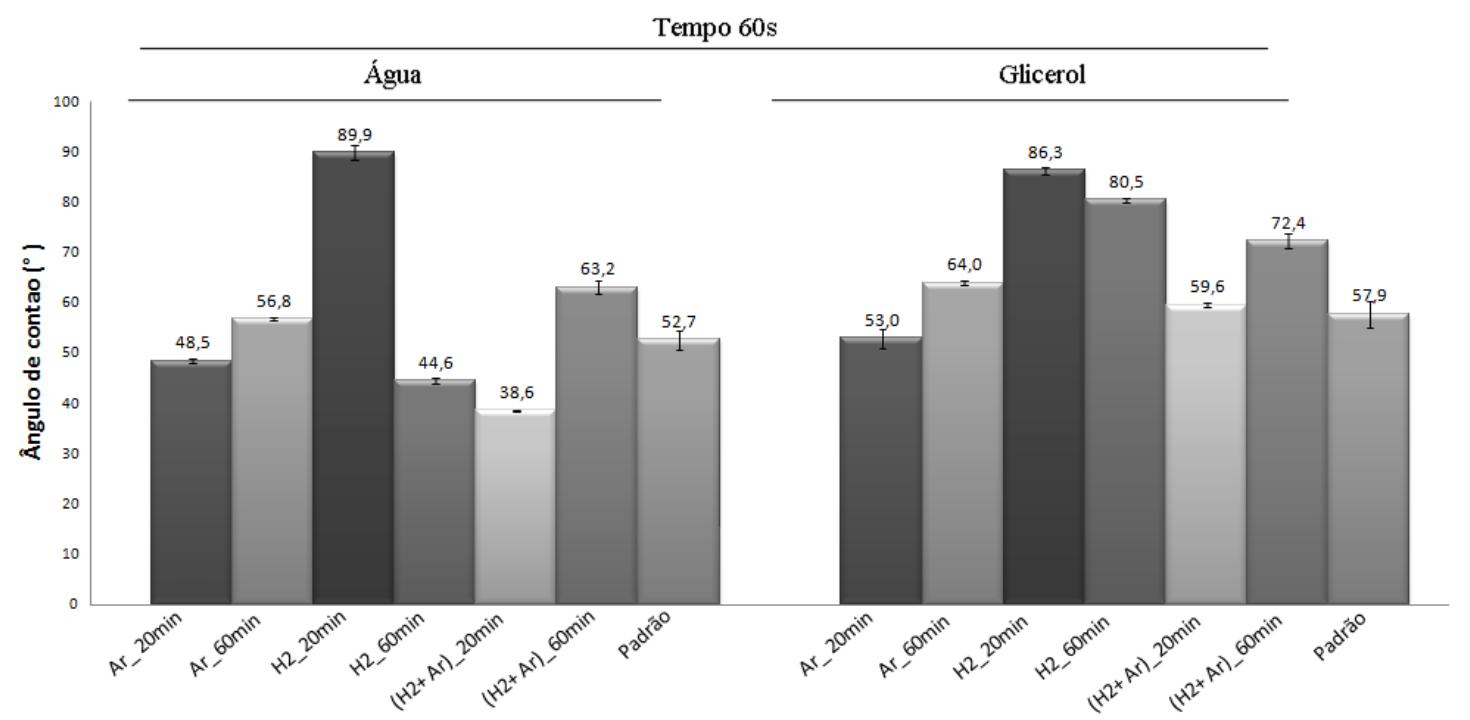

Figura 5 - Valores do ângulo de contato de uma gota de água (5a) e glicerol (5b) em superfícies de titânio tratadas por plasma

Nesta figura 5 pode-se observar que as duas maiores variações ocorridas foram para as condições $\mathrm{H}_{2} 60$ e $\mathrm{Ar} / \mathrm{H}_{2}$ 20. Nestas condições observa-se um aumento no valor do ângulo de contato de $44,60^{\circ}$ para $86,30^{\circ}$ (amostra $\mathrm{H}_{2} 60$ ) e de $38,60^{\circ}$ para $59,60^{\circ}$ (amostra $\mathrm{Ar} / \mathrm{H}_{2}$ 20) quando muda da água para o glicerol, respectivamente. Uma das hipóteses para explicar a grande diferença nesses valores pode ser feita com base no ângulo de contato aparente dado pela equação Cassie - Baxter modificada (eq. 1), introduzida por Marmur ${ }^{20}$.

$$
\cos \theta^{*}=r \phi_{s} \cos \theta+\phi_{s}-1
$$

Onde $\theta^{*}$ é o ângulo de contato aparente quando a superfície é rugosa, $\mathrm{r}$ é a razão entre a superfície real e a superfície geométrica, $\theta$ é o ângulo de contato de uma superfície lisa e $\phi_{\mathrm{s}}$ é a fração da superfície molhada pelo líquido. Quando uma gota é depositada 
sobre uma superfície rugosa haverá uma acomodação do líquido que dependerá da tensão superficial líquido-ar e ângulo de inclinação da superfície do vale. A figura 6 ilustra essa situação.

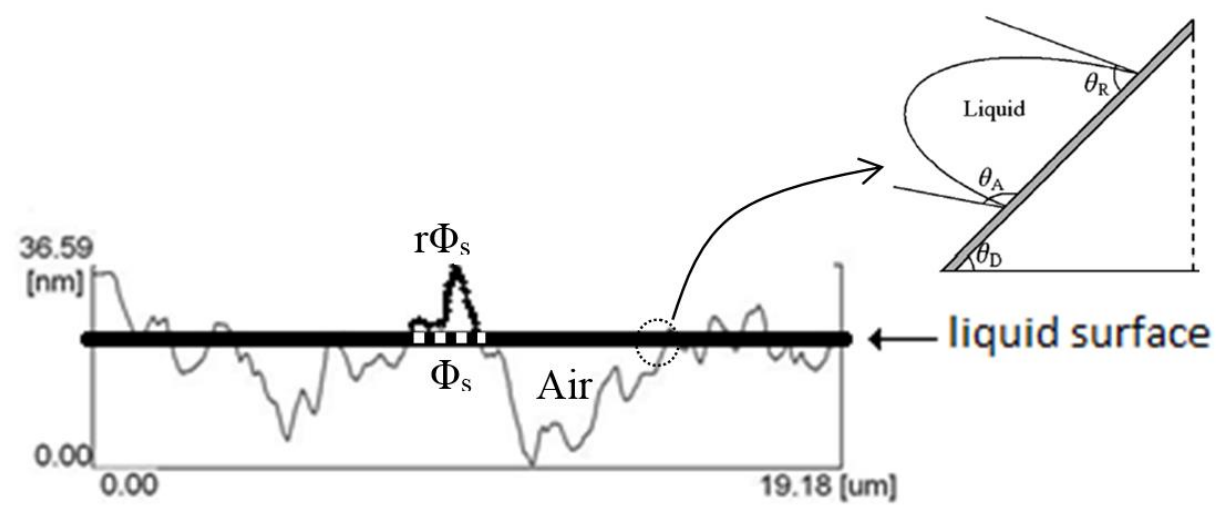

Figura 6 - Esquema ilustrativo da influência da tensão superficial e inclinação da superfície

Como a tensão superficial glicerol-ar é menor que da água-ar, significa que haverá uma maior tendência de expulsão para a gota do glicerol devido ao ar presente nos vales. As superfícies possuindo maior número de vales na região ocupada pela gota terão maiores diferenças do ângulo de contato aparente entre a água e o glicerol. Na figura 7 são mostrados os perfis topográficos extraídos das análises por AFM das amostras estudadas.

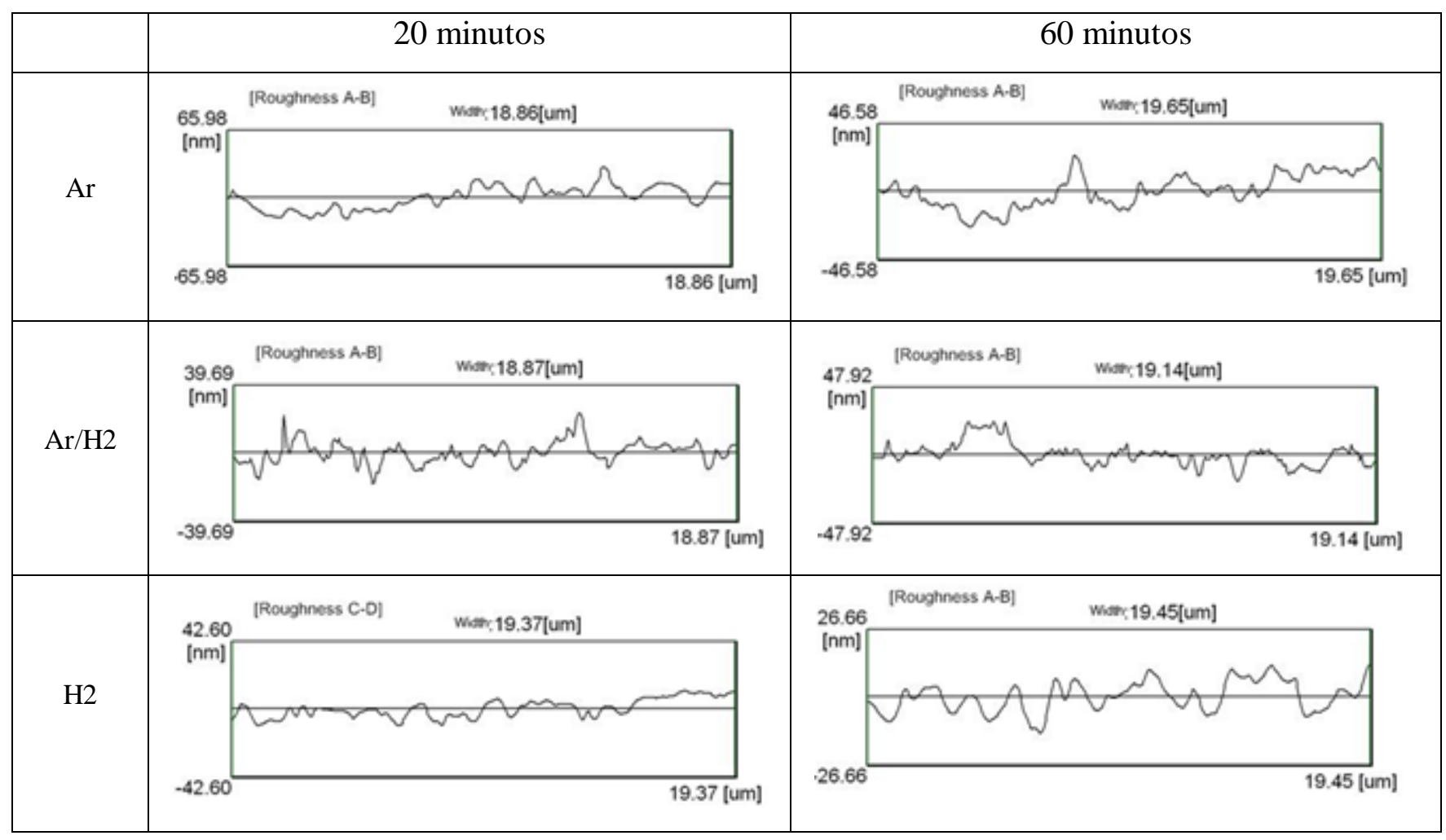

Figura 7 - Perfis topográficos das amostras com diferentes parâmetros de tratamento 
Pode-se observar que essas superfícies possuem diferenças de perfis, apesar dos valores de $\mathrm{Ra}$ e/ou $\mathrm{Rp} / \mathrm{Rz}$ em alguns casos serem próximos. Por exemplo, as amostras $\mathrm{Ar} / \mathrm{H}_{2} 60$ e $\mathrm{H}_{2} 60$ apesar de possuírem valores de $\mathrm{Ra}$ e $\mathrm{Rp} / \mathrm{Rz}$ muito próximos, os perfis topográficos são bastante diferentes.

Da mesma forma pode ser observada na amostra Ar 20, apesar de ter uma razão $\mathrm{Rp} / \mathrm{Rz}$ maior que 0,5 ela apresentou menor angulo de contato (maior molhabilidade), enquanto que o contrário aconteceu na amostra $\mathrm{Ar} / \mathrm{H}_{2}$ 60. Em uma observação mais atenta dos perfis nanotopograficos, verifica-se que essa última apresenta conjunto de picos com menor distância entre si, aumentando o ângulo de contato aparente, justificando assim a menor molhabilidade da amostra Ar/H2 $60 \mathrm{~min}^{21,22}$.

Desse modo, esses parâmetros de rugosidade discutidos no presente trabalho ainda não são suficientes para se realizar correlações diretas entre a topografia da superfície e a molhabilidade. É preciso um parâmetro que leve em consideração valores de $r$ e $\phi_{\mathrm{s}}$ apresentados na equação de Cassie-Baxter (eq. 1) ${ }^{22}$.

Um dado interessante de correlação entre molhabilidade e topografia da superfície acontece quando se compara os valores médios da razão $\mathrm{Rp} / \mathrm{Rz}$ com o ângulo de contato, independente da condição de tratamento. A figura 8 mostra um gráfico da $\mathrm{Rp} / \mathrm{Rz} \mathrm{em}$ função do ângulo de contato para água. Como pode ser observado, há uma correspondência direta entre os mesmos, quando se observa as superfícies com picos pontiagudos separadamente das superfícies dom picos arredondado.

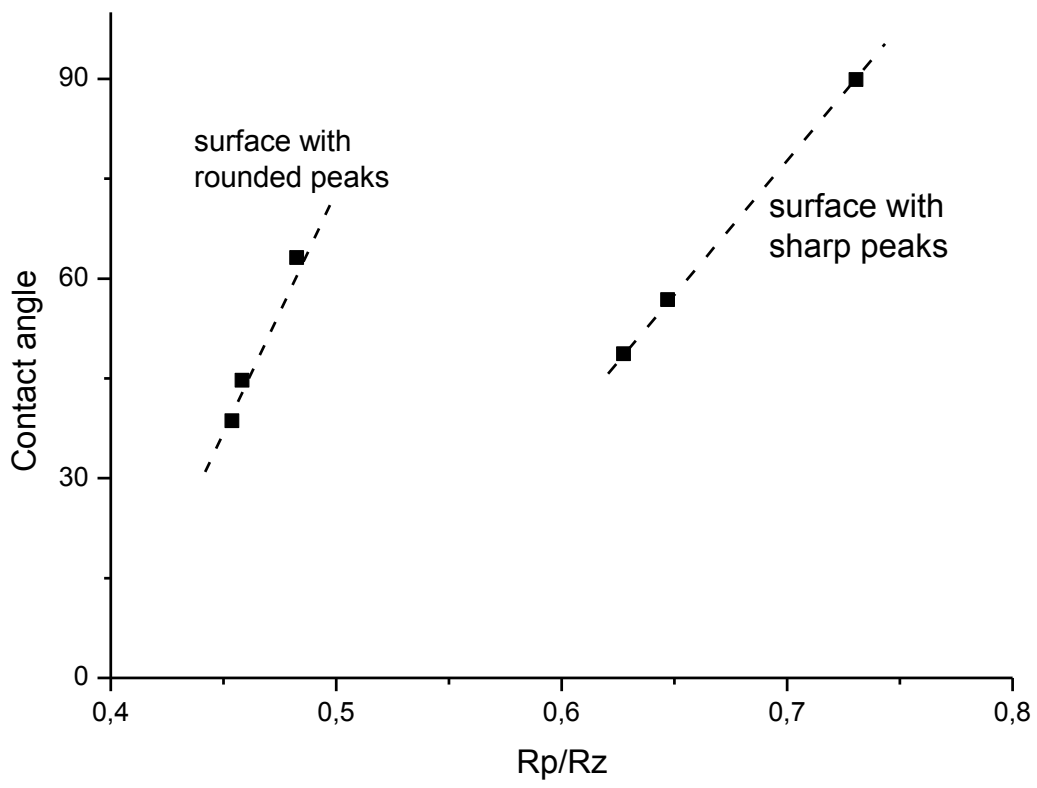

Figura 8 - Gráfico do ângulo de contato com a razão entre $\mathbf{R p} / \mathbf{R z}$.

\section{CONCLUSÃO}

O presente trabalho discute a correlação entre topografia de superfície e molhabilidade, mostrando que os parâmetros convencionais de rugosidade até o momento apresentados para correlacionar não são suficientes. Nesse sentido é sugerido um novo parâmetro que leve em consideração termos contidos na equação de Cassie Baxter, como a distância entre picos e r $\phi_{\mathrm{s}}$. Também é mostrado que processos 
preliminares como a limpeza de superfície, pode resultar em diferentes valores de molhabilidade. Dos resultados obtidos pode-se concluir que:

1. Tratamentos de limpeza efetuados para remoção de óxidos foram efetivos para todas as condições de plasma estudadas, ou seja, argônio puro, hidrogênio puro e mistura argônio-hidrogênio.

2. Todas as condições apresentaram um padrão de DRX semelhante, contendo principalmente Ti $\alpha$ e pequena quantidade de óxido $\mathrm{Ti}_{2} \mathrm{O}$, obtido após redução do $\mathrm{TiO}_{2}$.

3. Não foi observada correlação entre o parâmetro Ra e a molhabilidade, nem mesmo entre pares com a mesma atmosfera e tempos de tratamento diferentes.

4. Foi observada uma correlação entre o parâmetro $\mathrm{Rp} / \mathrm{Rz}$ com a molhabilidade, quando comparadas aos pares tratadas com a mesma atmosfera, mas a proporcionalidade não foi direta.

5. Quando as superfícies foram agrupadas por grupos com $\mathrm{Rp} / \mathrm{Rz}$ menor que 0,5 (picos arredondados) e $\mathrm{Rp} / \mathrm{Rz}$ maior que 0,5 (picos pontiagudos) houve correlação com proporcionalidade direta.

6. Há necessidade de um novo parâmetro topográfico que leve em consideração os termos contidos na equação de Cassie - Baxter, como a distância entre picos e r $\phi_{\text {s. }}$.

\section{AGRADECIMENTOS}

Ao LAIS - Laboratório de Inovação Tecnológica em Saúde e ao LAPLASMA da Universidade Federal do Rio Grande do Norte.

\section{REFERÊNCIAS BIBLIOGRÁFICAS}

[1] BINON, P.P.; WEIR, D.J. MARSHALL, S.J. Surface analysis of an original Branemark implant and three related clones. Int. J. oral Maxillofac. Implants, Summer 1992. 7n. 2, p. 168-175.

[2] KASEMO, B. Surface Science. 2002; 500, 656

[3] KASEMO, E., LAUSMAA,J.: Biomaterial and implant surfaces: On the role of cleanliness, contamination and preparation procedures. J. Biomed. Mater .Res. 1988; 22:1.45-1.58.

[4] M. TAKEUCHIA et al., Acid pretreatment of titanium implants. Biomaterials, 2003; 24,1821-1827.

[5] LIU X., CHU P. K., DING C., Surface modification of titanium, titanium alloys, and related materials for biomedical applications, Materials Science and Engineering, 2005;01 - 69.

[6] CHANG H.Y. el al., Surface Oxide that cleans for a Surface of plasma of atmospheric pressure and Layers, Materials Science and Engineering, 2004; 177 $-178,711-715$.

[7] SÁ, J. C., et al. Influence of argon ion bombardment of titanium surfaces on the cell behavior. Surface \& Coatings Technology. 2008; 203: 1765-70.

[8] SILVA, M. A. M.; MARTINELLI, A. E.; ALVES JR, C.; NASCIMENTO, R. M.; TÁVORA, M. P.; VILAR, C. D. Surface modification of Ti implants by plasma oxidation in hollow cathode discharge. Surface \& Coatings Technology 20, 612 620,2005 . 
[9] GUERRA NETO, C.L.B; SILVA, M.A.M,; ALVES, JR C., In vitro study of cell

[10] behaviour on plasma surface modified titanium. Surface Engineering, p.146$150,2009$.

[11] GUERRA NETO, C. L. B., SILVA, M. A. M., ALVES JR., C. Experimental study of plasma nitriding dental implant surfaces. Surface Engineering. 2009; 25,1-4.

[12] HSIEH J.H., C. LI. Effects of hollow cathode and $\mathbf{A r} / \mathbf{H}_{2}$ ratio on plasma cleaning of Cu lead frame. Surface and technology, 2006; 101-103.

[13] ZHU et al., Effects of topography and composition of titanium surface oxides on osteoblast responses, Biomaterials, 2004; 25, 4087- 4103.

[14] GUERRA NETO, C. L. B. ; SILVA, M. A. M. ; ALVES JR, C. . Osseointegration evaluation of plasma nitrided titanium implants. Surface Engineering, v. 25, p. 434-439, 2009

[15] ALVES JR, C. et al., Nitriding of titanium disks and industrial dental implants using hollow cathode discharge. Surface and Coatings Technology, 2005; 194, Issues 2-3:196-202.

[16] WHITEHEAD SA, SHEARER AC, WATTS DC, WILSON NH. Comparison of methods for measuring surface roughness of ceramic. J Oral Rehabilitation 1995; 22(6): 421-7.

[17] ELIAS, C. N., OSHIDA, Y., LIMA, J. H. C et al. Relationship between surface properties (roughness, wettability and morphology) of titanium and dental implant removal torque. Journal on Mechanical Behavior of Bio medical Materials, v. 1, p. 234-42, 2008.

[18] LIM, Y. J., OSHIDA, Y. Initial contact angle measurements on variously treated dental/medical titanium materials. Biomedical materials and engineering, p.1-17, 2001

[19] ALBREKTSSON, T., WENNERBERG, A. Oral implant surfaces: Pat 1 - review focusing on topographic and chemical properties of different surfaces and in vivo responses to them. International Journal of Pros-thodont v. 17 , p. 536-43, 2004.

[20] CHRISTENSON EM, ANSETH KS, VAN DEN BEUCKEN JJ, CHAN CK, ERCAN B, JASEN JA et al. Nanobiomaterial applications in orthopedics. J Orthop Res 2007;25:11-22.

[21] A. MARMUR, LANGMUIR 19 (2003) 8343-8348.

[22] H. NAKAE, R. INUI, Y. HIRATA, H. SAITO, Effect of surface roughness on wettability, Acta Mater. 46, 7 (1998) 2313-2318.

[23] Y.Y. YAN.; N. GAO.; W. BARTHLOTT. Mimicking natural superhydrophobic surfaces Bormashenko, E., General equation describing wetting of rough surfaces, Journal of Colloid and Interface Science 360 (2011) 317-319 